Service social

\title{
Les voies non verbales du travail social, textes présentés au VIe congrès du groupe régional européen de l'Association internationale des écoles de service social, publiés par l'École supérieure d'action sociale de Liège, Belgique, 1991, 204 pages.
}

\section{Christian Côté}

Volume 41, numéro 2, 1992

Bilan des réformes

URI : https://id.erudit.org/iderudit/706576ar

DOI : https://doi.org/10.7202/706576ar

Aller au sommaire du numéro

Éditeur(s)

École de service social de l'Université Laval

ISSN

1708-1734 (numérique)

Découvrir la revue

Citer ce compte rendu

Côté, C. (1992). Compte rendu de [Les voies non verbales du travail social, textes présentés au VIe congrès du groupe régional européen de l'Association internationale des écoles de service social, publiés par l'École supérieure d'action sociale de Liège, Belgique, 1991, 204 pages.] Service social, 41(2), 167-169. https://doi.org/10.7202/706576ar d'utilisation que vous pouvez consulter en ligne. 
une bonne synthèse des connaissances sociales, juridiques et psychologiques dans le domaine de la médiation. Les personnes qui divorcent, tout comme les professionnels qui interviennent dans ce domaine et les étudiants, y trouveront une source de références précieuse, faisant le point sur cette pratique.

Madeleine BEAUDRY École de service social Université Laval

\section{LES VOIES NON VERBALES DU TRAVAIL SOCIAL}

Textes présentés au $\mathrm{Vl}$ e congrès du groupe régional européen de l'Association internationale des écoles de service social, publiés par l'École supérieure d'action sociale de Liège, Belgique, 1991, 204 pages.

Le titre de l'ouvrage m'avait amené à croire qu'il s'agissait d'une analyse des moyens et canaux non verbaux de la communication interpersonnelle et intergroupes. J'ai eu l'agréable surprise de découvrir un contenu fort différent, mais aussi intéressant que ce à quoi je $\mathrm{m}^{\prime}$ attendais. Dans la ligne de plusieurs approches européennes en travail social, Les voies non verbales du travail social présente des analyses théoriques et des expériences d'interventions sur les cadres de la vie quotidienne. Son titre indique une volonté de se démarquer des approches trop exclusivement verbales, empruntées à la psychothérapie et aux approches récentes en communication interpersonnelle. En même temps, la plupart des auteurs soulignent que la communication verbale en est augmentée et enrichie.

Présenté par le Dr Lopez, directeur de l'ESAS, I'ouvrage se compose de seize textes soumis au $\mathrm{Vl}^{\mathrm{e}}$ congrès de $\mathrm{l}^{\prime}$ Association internationale des écoles de service social par des praticiens-chercheurs et des professeurs de service social de sept pays. Les auteurs insistent sur l'organisation nouvelle et l'utilisation systématique des milieux de vie. Leur postulat est que le milieu de vie quotidienne constitue un cadre qui, au-delà des mots, développe une perception et un décodage qui lui sont propres. Ce cadre influence les échanges de valeurs, d'idées, de sentiments, et amène les personnes qui y vivent à développer de nouvelles significations des situations vécues.

Un groupe d'articles décrivent des expériences d'organisation de vie et d'activités, avec les groupes marginaux, les décrocheurs, les délinquants, les handicapés physiques et mentaux. Ils présentent des expériences d'appartements supervisés, de stage de travail ou de sport, d'intervention à domicile au lieu de cures de désintoxication. Casado souligne que même s'il y a de nombreux échanges verbaux avec les usagers, les principaux échanges se font avec les milieux, pour les amener à une organisation nouvelle et à un encadrement approprié. Dutrenit souligne qu'à l'établissement de fortes exigences, accompagnées de fortes récompenses, est habituellement reliée une amélioration sensible des comportements des usagers. 
Des articles approfondissent les modalités de l'apprentissage par l'action, soit pour les bénéficiaires de services : Lopez, Casado, Heiner, Fosteny et Rossel, soit pour les étudiants en service social : Wieler, Dutrenit, Graitson, Chetkow, Espelund, Anciaux, etc. Les textes relient les théories de I'apprentissage, de la cognition et de l'action sociale de façon très intéressante. Fosteny et Rossel soulignent qu' "en se centrant sur un aspect linguistique au lieu de stimuler une activité, on contribue à retarder la capacité de progresser» et on peut «provoquer un besoin d'approbation sociale» (p. 49). Tandis que «proposer une réalisation d'activités plutôt qu'une verbalisation de ces activités permet l'accès à une régulation basée sur le résultat obtenu, ce qui permet de passer à une nouvelle activité à partir de la première réussie» (p. 150).

Près de la moitié de ce groupe de textes sont écrits par des professeurs. Ils présentent des expériences d'enseignement, de méthodologie, de supervision et de stages. Ils insistent sur la nécessité des apprentissages agis, vécus en partage avec les clients. Wieler souligne l'équilibre ainsi atteint, alors que "la sophistication intellectuelle tend à écraser le sentiment et I'action; I'activisme occulte la réflexion et l'évaluation; et une émotivité extrême pourra bloquer la pensée et I'action...» (p. 132). Graitson et d'autres font un lien avec la praxis de Paulo Freire, en insistant sur la qualité de la connaissance de l'autre et de la responsabilité acquises par la coopération, le travail en commun, le partage des mêmes idées et des mêmes émotions dans la même situation. Graitson rappelle qu'une excellente méthode non verbale de résolution des conflits est de faire des activités agréables ensemble...

Enfin, on trouve dans plusieurs acticles des techniques d'intervention non verbale - même si elles sont accompagnées de verbalisations : le cahier de mémoires (photos) - les cartes de compétences interpersonnelles et sociales les grilles de comportements - le «thermomètre des sentiments» - les dessins les bandes dessinées - l'utilisation des jouets et des objets quotidiens significatifs - le modelage des émotions et la sculpture familiale - le génogramme la carte écologique - les diaporamas - les vidéos - les rapports écrits, etc. Les articles de Heiner, Elliott et $A$. Mullender, déjà connue au Québec pour son approche de groupe, sont spécialement intéressants.

Des articles font voir l'utilisation du ballet pour les handicapés (Casado) ; la pièce de théâtre pour amener les étudiants à prendre conscience que les décisions - celles des gestionnaires et les leurs - sont liées à des positions idéologiques habituellement cachées ou non conscientes (Anciaux) ; la peinture pour développer un sens d'identité personnelle et communautaire (Mahy et Seret) ; le sport auprès de jeunes marginalisés (De Beer).

Les voies non verbales du travail social constitue un ouvrage non seulement intéressant, mais stimulant. Le fait de découvrir des réflexions et des expériences d'auteurs de plusieurs pays et de qualité généralement élevée est rafraîchissant et soutenant. II montre que des expériences que nous avons peut-être déjà pensé tenter, mais qui dérangeaient les façons établies et demandaient donc un effort spécial, ont été réalisées par d'autres, et non seulement avec efficacité pour les clients, mais aussi avec des gratifications pour les professionnels. II souligne que le changement vient souvent des actions réalisées avec ou par les clients, en leur laissant beaucoup de place. 
Je recommande spécialement cette lecture aux professionnels découragés qui croient qu'en désespoir de cause mieux vaut parler de changer ou d'apprendre que de faire des activités... D'ailleurs, Eriksson soulève l'hypothèse que l'utilisation du pouvoir, légal ou illégal, peut être liée au désespoir de l'intervenant... Heiner propose de son côté que remettre par des moyens concrets le pouvoir de s'auto-évaluer aux clients, aux groupes, aux familles diminue le poids sur les épaules du professionnel.

Plusieurs des expériences rapportées sont marginales et ne suivent pas les règles habituelles. Mais leurs objectifs minimaux constituent peut-être une raison de leur efficacité apparente : faire "plus du même» avec des marginaux amène «plus du même», c'est-à-dire la répétition de l'échec, en dépit de la qualité des interventions.

Les articles ne sont pas tous de valeur égale. Quelques-uns sont écrits dans un style dense. Plusieurs conclusions très positives ne sont pas encore étayées par des recherches sérieuses, comme c'est généralement le cas dans l'état actuel de la science en service social. Mais la lecture est profitable à ceux qui veulent chercher des voies innovatrices et utiliser les contextes de la vie quotidienne. Il serait intéressant de faire des liens entre les propositions de l'ouvrage et les expériences menées dans d'autres pays, dont le Québec, et particulièrement avec l'approche dite communautaire actuellement en développement dans les CLSC, afin de l'éclairer et de l'enrichir.

Christian CÔTÉ

École de service social Université Laval

\section{RECHERCHES ÉVALUATIVES EN TRAVAIL SOCIAL}

François Le Poultier,

Presses universitaires de Grenoble, France, 1990, 249 pages.

Dès le départ, l'auteur nous situe sur l'objectif de son ouvrage qui est « de montrer qu'une recherche évaluative menée dans le travail social en référence à la méthode expérimentale est non seulement possible mais qu'elle est la forme d'investigation la plus appropriée pour régler actuellement l'obsédante question de I'évaluation » (p. 13). Cet ouvrage tente donc de réconcilier travailleurs sociaux et expérimentalistes sur la base de préoccupations professionnelles liées à l'évaluation.

Les six chapitres composant cet ouvrage se divisent en deux blocs : alors que les trois premiers chapitres se veulent plus théoriques, les trois derniers racontent des expériences d'évaluation dans le domaine de l'assistance sociale et de l'éducation spécialisée.

Sur le plan théorique, un premier chapitre traite du travail social et de son évaluation. On y présente le travail social comme « un ensemble professionnel mou », non homogène, intervenant auprès de clientèles très diverses, et où la culture psychologique domine. En même temps que l'on voit se développer, en 